\title{
From ignition to stable combustion in a cavity flameholder studied via 3D tomographic chemiluminescence at $20 \mathrm{kHz}$
}

\author{
Lin Ma, ${ }^{1,2, *}$ Qingchun Lei, ${ }^{2}$ Yue Wu,${ }^{1}$ Wenjiang Xu, ${ }^{1}$ Timothy M. Ombrello, ${ }^{3}$ \\ Campbell D. Carter \\ ${ }^{1}$ Department of Aerospace and Ocean Engineering \\ Virginia Tech, Blacksburg, VA 24060, USA \\ ${ }^{2}$ Department of Mechanical Engineering \\ Virginia Tech, Blacksburg, VA 24060, USA \\ ${ }^{3}$ Air Force Research Laboratory \\ Wright-Patterson AFB, OH 45433, USA \\ *Corresponding author: linma@vt.edu \\ Laser Diagnostic Lab \\ Room 660A McBryde Hall \\ Department of Aerospace and Ocean Engineering \\ Virginia Tech \\ Blacksburg, VA 24060, USA \\ Phone: 1(540)231-2249
}

Fax: 1(540) 231-9632

Page 1 of 29 


\begin{abstract}
This work reports the study of the ignition processes in a Mach-2 cavity combustor based on three-dimensional (3D) measurements at $20 \mathrm{kHz}$. The 3D measurements were obtained by a combination of tomographic chemiluminescence and fiber-based endoscopes. Measurements of 3D flame and flow properties were reported under two fueling conditions of the combustor. The properties included 3D volume, surface area, shape factor, and 3D3C (three-dimensional and three-component) velocity of the ignition kernel. These results clearly distinguished the ignition stage from the stable combustion stage of the combustor and enabled the determination of a transition time to quantify both stages. The analysis of the change of the ignition kernel's shape, when combined with the 3D3C velocity measurements, also illustrated flame-flow interactions in the cavity combustor. These results demonstrated the utility of the $3 \mathrm{D}$ diagnostics to overcome some of the limitations of established planar diagnostics and to resolve the dynamics of highspeed combustion devices both spatially and temporally.
\end{abstract}

\title{
Keywords
}

3D measurements, tomography, fiber based endoscopes, supersonic combustion 


\section{Introduction}

One of the key challenges to the development of supersonic air-breathing systems is the design of reliable ignition and flame stabilization techniques. However, such processes are highly dynamic and involve the intricate interactions of several complex processes that are not fully understood yet $[1,2]$. As a result, a significant amount of research, both computational [3-5] and experimental [6-8], has been invested for a better understanding of the ignition and flameholding processes at a fundamental level and also for the design of practical devices.

This work reports an experimental study of the ignition processes in supersonic (Mach 2) flows using three-dimensional (3D) measurements obtained via tomographic imaging of chemiluminescence. Non-intrusive optical techniques are usually preferred or required for the experimental study of ignition and flameholding processes in supersonic flows, and a range of optical diagnostics have been developed and employed in past efforts, which can be broadly divided into two categories. The first category of experimental techniques rely on line-of-sight measurements such as chemiluminescence imaging [9], schlieren imaging [7], shadowgraph imaging [9], or tunable laser absorption spectroscopy [10]. Techniques in this category enjoy the advantage of being relatively simple in terms of implementation, yet able to provide a range of key properties including development of the ignition kernels, flame propagation, temperature, and concentration of chemical species. Furthermore, it is straightforward to obtain measurements with high temporal resolution because of the commercial availability of high-speed cameras and detectors. The limitations of these techniques are their line-of-sight nature, which results in a lack of spatial resolution along an integrated path to resolve the structures of the ignition and flameholding processes. The second category of experimental techniques relies on laser planar imaging measurements, such as PLIF (planar laser-induced fluorescence) $[1,11,12]$ and PIV (particle imaging velocimetry) $[8,13]$. Techniques in this category provide measurements with well-defined spatial resolution across a 2D plane of key combustion and flow properties. Using these techniques, instantaneous planar measurements of combustion radicals and intermediates (e.g., $\mathrm{OH}, \mathrm{CH}_{2} \mathrm{O}$ ) and velocity fields have been performed to study the flameholding, flame propagation, and mixing in supersonic combustors. The limitations of these techniques include their relatively low repetition rate and their $2 \mathrm{D}$ nature. The lasers used for these measurements typically operate at a repetition rate on the order of $10 \mathrm{~Hz}$, which is not sufficient to resolve the 
temporal evolution of the ignition and flame stabilization processes. However, recent development of high-speed pulsed lasers is poised to enable 2D PLIF and PIV measurements in supersonic flows with dramatically improved repetition rate [14-16]. Nevertheless, the planar images obtained from these techniques provide a 2D slice of a complicated 3D structure, which is generally insufficient to infer the target flow or flame properties conclusively.

Diagnostic techniques that can resolve the 3D spatial flame and flow structures with sufficient temporal resolution are ultimately needed to resolve the fundamental processes involved in supersonic combustion. Ongoing research is focusing on pursuing such diagnostic capability actively. For example, recent efforts have demonstrated that the combination of tunable laser absorption spectroscopy and tomography can overcome the line-of-sight limitation of the traditional absorption technique to obtained spatially resolved data. Measurements have been successfully demonstrated in a scramjet combustor [17] and a practical gas turbine engine [18] at rates up to $50 \mathrm{kHz}$. Progress to extend PLIF and PIV techniques for high-speed 3D measurements has also been reported. Examples include the PLIF measurements at several locations by scanning the excitation laser sheet at $\sim 1 \mathrm{kHz}[19,20]$, and 3D three-component velocity measurements by combining PIV with tomography [21-23].

In this work, we report the study of the ignition processes in a cavity flameholder within a Mach-2 crossflow using 3D measurements at $20 \mathrm{kHz}$ obtained from tomographic chemiluminescence (TC). The measurements were made in the Research Cell 19 supersonic wind-tunnel facility housed at the Air Force Research Laboratory (AFRL) [24]. The TC diagnostic combines chemiluminescence with tomography to overcome the line-of-sight limitation of traditional chemiluminescence imaging [25, 26]. More specifically, with the TC diagnostic we have measured line-of-sight cavity chemiluminescence images (termed projections) simultaneously from 8 different orientations, each at a rate of $20 \mathrm{kHz}$. These projections were then fed into a tomographic reconstruction algorithm [27] as inputs to obtain 3D measurements of combustion processes. Processing the tomographic reconstruction frame by frame resulted in a sequence of 3D measurements at a rate of $20 \mathrm{kHz}$. Further analysis of these measurements were then performed to obtain quantities of key interest to the study of the ignition processes, including the temporal evolution of the 3D topography, position, volume, surface area, and velocity of the ignition kernels under different conditions. 
This work also illustrates the practical utility of fiber-based endoscopes (FBE) in 3D optical diagnostics. Obtaining tomographic measurements (either via tomographic chemiluminescence, PIV, or absorption $[17,18,25,28]$ ) requires projection measurements from multiple viewing angles and locations. Furthermore, these projections must be obtained simultaneously for temporally resolved measurements, thusly requiring multiple high-speed cameras or detectors. Such requirements pose practical challenges in terms of optical access, physical space, and equipment cost. Investigation in recent years has demonstrated the use of FBEs to be an effective solution to these practical issues [29-31]. As illustrated in this work, FBEs have a relatively small footprint ( $\sim 2 \mathrm{~cm}$ fiber core), when compared to a standard highspeed camera, and they are flexible enough to be installed when optical access and physical space are limited. Furthermore, customization of the FBEs allowed for the recording of four projections onto a single camera, significantly reducing the equipment cost and implementation difficulty.

In the rest of this paper, Section 2 outlines the experimental setup, including details of the supersonic combustor, testing conditions, as well as the hardware used to in the TC diagnostic. Section 3 describes the 3D tomographic reconstruction methods and the validation results, followed by a discussion of the 3D properties (i.e., topography, position, volume, surface area, and velocity) of the ignition kernels in Section 4. Section 5 then summarizes and concludes the paper.

\section{Experimental Arrangement}

The experimental setup is illustrated schematically in Figure 1. The experiments were performed in a supersonic wind tunnel housed in Research Cell 19 at AFRL [8, 9]. The facility is capable of operating continuously with peak stagnation conditions of $2,860 \mathrm{kPa}$ and $922 \mathrm{~K}$ at flow rates up to $15.4 \mathrm{~kg} / \mathrm{s}[24,32]$. Figure 1a illustrates the overall experimental setup from the end view (with the Mach-2 flow issuing into the paper), Figure $1 \mathrm{~b}$ the side view of the wind tunnel and the cavity combustor, and Figure $1 \mathrm{c}$ the top view of the cavity. The direction of the Mach-2 flow $(M=2)$ was from left to right in both Figure $1 \mathrm{~b}$ and $\mathrm{c}$, as shown. The entire flow path is $152 \mathrm{~mm}$ wide. There are two ports in the base of the cavity located $19 \mathrm{~mm}$ on either side of the symmetry plane to accommodate spark plugs [8]. The two spark plugs (represented by the star symbols) were of the typical automotive style with a center high voltage electrode and 
surrounding ground. Excitation for each plug was provided by a transistorized coil system (AEM part number 30-2853) with energy deposition of up to $100 \mathrm{~mJ} / \mathrm{pulse}$. The two sparks were fired simultaneously, and served as the sole means to ignite the mixture. Control electronics were used to synchronize the operation of the cameras and spark plugs as shown in Figure 1a. Fuel $\left(\mathrm{C}_{2} \mathrm{H}_{4}\right)$ was injected into the cavity from eleven holes in the cavity closeout ramp as shown in Figure 1b; it should be noted that $\mathrm{C}_{2} \mathrm{H}_{4}$ was used primarily because it is a single-component fuel (and thus easy to prepare) having high reactivity. The total temperature and pressure (i.e., the set values upstream of the $2 \mathrm{D}$ nozzle) for all experiments were approximately $610 \mathrm{~K}$ and $483 \mathrm{kPa}$, respectively, while the measured static pressure within cavity was about $63 \mathrm{kPa}$ before ignition and $69 \mathrm{kPa}$ after the cavity reaches quasi-steady burning. There was optical access from both the side and top through fused-silica windows, allowing examination of the cavity ignition and burning process from multiple views as shown in Figure 1a.

Chemiluminescence was captured from eight orientations simultaneously using eight FBE inputs and two CMOS (complementary metal-oxide semiconductor) cameras (Photron SA$Z$ ). The use of eight FBEs (not more) was due to the constraint of the optical access available. The FBEs used here were customized and described in detail in $[30,31,33]$. These FBEs were provided as two customized bundles, with four inputs per bundle. The four inputs in each bundle are then combined into one output, so that chemiluminescence images captured by all four inputs can be captured by one camera. More specifically, as Figure 1a schematically shows, camera 1 captured the chemiluminescence images from FBE input 1 to 4 of bundle 1, and camera 2 the images from FBE inputs 5 to 8 of bundle 2 . A lens ( $105 \mathrm{~mm}$ focal length and $\mathrm{f} / 2.8)$ was placed in front of each FBE input to enlarge the collection angle. Each FBE input consists of an array of $470 \times 470(220,900)$ individual single mode fibers, and therefore the output of each bundle transmits a total of $883,600(4 \times 220,900)$ image elements to the camera, which operated at a pixel resolution of $1024 \times 1024$. The FBE bundles and the cameras were aligned in such a way that one image element transmitted by the FBE approximately corresponded to one pixel on the camera [34]. Both cameras were operated at a frame rate of $20 \mathrm{kHz}$ and an exposure time of $49 \mu \mathrm{s}$. The number of pixels read out decreases as the frame rate increases beyond $20 \mathrm{kHz}$. Therefore, even though a higher temporal resolution is desired to better follow the dynamics of the target combustion processes, this work was performed at $20 \mathrm{kHz}$ for the consideration of the resolution (and/or field of view), and the approximate 1:1 matching between FBE image elements and the 
pixels as aforementioned. Before any measurement, a calibration target was placed in the cavity to determine the orientation and location of the FBEs using a view registration program [30]. All 8 FBE inputs were aligned in the plane perpendicular to the flow (i.e., the $Y Z$ plane as shown), and their orientation (defined as the angle formed relative to the $Z$ axis) was $180^{\circ}, 169^{\circ}, 144^{\circ}, 92^{\circ}$, $89^{\circ}, 38^{\circ}, 18^{\circ}$, and $0^{\circ}$, respectively, for FBE inputs 1 through 8 .

Experiments were performed under various conditions, and this work reports results obtained with fuel flow rates of 56 and 99 standard liters per minute (SLPM). These fuel flow rates, respectively, represent approximately the lowest and highest fuel flow rates at which ignition can be initiated in the combustor with the sparks in their current configuration. It is our hypothesis (based on simulations and recent measurements) that this range of fuel flow rates relates to a range of flammability (for $\mathrm{C}_{2} \mathrm{H}_{4}$-air at the specific static temperature and pressure) in the vicinity of the spark igniters. With each fuel flow rate, 11 experiments were performed under the same conditions. Figure 2 shows two sample chemiluminescence projections captured by camera 2 for one of the low fueling rate experiments to better illustrate the nature of the diagnostics and the operation of the experiments. Sparks were initiated about $9 \mathrm{~ms}$ after charging began. Time zero $\left(t_{0}\right)$ is then defined as the time that a spark first appears on the camera, and the discharge of the sparks lasted for $\sim 3-4 \mathrm{~ms}$. The cameras began framing at the same time that the spark plugs began to charge, and chemiluminescence images were captured for a duration of 50 $\mathrm{ms}$ (resulting in a total of 1,000 frames) per experiment. This duration was sufficiently long to capture the entire ignition and flame stabilization processes (if the experiment resulted in a successful ignition). Figure $2 \mathrm{a}$ shows the projections captured by camera 2 at $t=0.8 \mathrm{~ms}$. As shown, the camera captured four projections transmitted by FBE inputs 5 through 8 simultaneously, with the projection transmitted by each FBE occupying approximately a quarter of the camera chip (i.e., $\sim 512 \times 512$ pixels). As mentioned, these measurements corresponded to views angle of $89^{\circ}, 38^{\circ}, 18^{\circ}$, and $0^{\circ}$, respectively. Therefore, the measurements transmitted through FBE input 5 provided a near top view of the cavity, and those through FBE input 8 a side view. Figure 2a shows that at $t=0.8 \mathrm{~ms}$, an ignition kernel had already developed while the sparks were still discharging (and also that both of the sparks were visible from the near top view provided by FBE input 5, and they overlapped from the side view provided by FBE 8 ). Figure $2 \mathrm{~b}$ shows the projections captured at $t=10 \mathrm{~ms}$, when a stable flame had been established in the combustor and the sparks had stopped discharging. 
Figure 3 illustrates more specifics of the operations of the experiments performed in this study. Figure $3 \mathrm{a}$ first defines several characteristic times to facilitate the description of the experiments and results. Again, time zero $\left(t_{0}\right)$ is defined as the time that the spark discharges initiated, which was experimentally determined by finding the first frame where emissions from the plasma created by the discharges was observed on the cameras. Figure $3 b$ shows such an example in the same low fueling rate experiment as in Figure 2. The measurements shown in Figure $3 \mathrm{~b}$ through $3 \mathrm{e}$ were all taken from this same experiment, from the side view by FBE input 8 (with an orientation of $0^{\circ}$ ) and from a view of $18^{0}$ by FBE input 7. Again the star symbols illustrate the locations of the spark plugs. The next characteristic time, $t_{1}$ as shown in Figure $3 \mathrm{a}$, was defined as the time that the ignition kernel initiated. Experimentally, $t_{1}$ was determined by finding the first frame where the emission from the kernel was observed on the cameras, and Figure $3 \mathrm{c}$ shows an example for which $t_{1}$ was determined to be $0.5 \mathrm{~ms}$. The third characteristic time, $t_{2}$ as shown in Figure 3a, was then defined as the time that the ignition kernel transitions into a stable flame. There is ambiguity in the determination of $t_{2}$, both in past work [7] and in this work. However, as to be discussed in Section 4, the availability of 3D measurements can help to better determine $t_{2}$ quantitatively (e.g., by examining the increase of the volume or surface area of the ignition kernel/flame). In this work, $t_{2}$ was defined as the time when the volume of the ignition kernel reached the average level of the stable flame. Figure $3 \mathrm{~d}$ shows an example for which $t_{2}$ was determined to be $3.5 \mathrm{~ms}$ in this case. The last characteristic time, $t_{3}$ as shown in Figure 3a, was defined as the time that the sparks stopped discharging. Experimentally, $t_{3}$ was determined by finding the frame after the last frame where the emissions from the spark discharges was observed on the cameras, and Figure $3 \mathrm{e}$ shows an example for which $t_{3}$ was determined to be $4.2 \mathrm{~ms}$.

Based on the above definition of the characteristic times, Table 1 summarizes all the datasets analyzed in this work. These datasets included two low fueling rate cases and two high fueling rate cases. A total of 22 datasets were gathered during this project (11 under the low fueling rate condition and another 11 under the high fueling rate condition). Each dataset contained a total of 2000 images captured for a duration of $50 \mathrm{~ms}$ (1000 images per camera). However, the chemiluminescence signal emitted by the ignition kernel was significantly weaker than that emitted by the sparks and the stable flame, as can be seen from Figure 2. As a result, the chemiluminescence signals from the ignitions kernel cannot always be determined reliably 
and continuously (i.e., in every frame of the data) for every dataset captured. After examining all 22 datasets, four datasets (those tabulated in Table 1) were identified to have continuous and unambiguous signals from the ignition kernel, and this work focused on the analysis of these four datasets only.

The data shown in Table 1 illustrated that $t_{0}$ was defined as the initial time where the emissions from the igniters were first observed, and $t_{3}$ was consistently near $4 \mathrm{~ms}$ because of the characteristic circuit discharge time of other exciters. The second and third row of Table 1 show that the low fueling rate cases exhibited earlier development of the ignition kernel and earlier transition into stable flames than the high fueling rate cases, as indicated by shorter $t_{1}$ and $t_{2}$, respectively. Table 1 shows that the $t_{1}$ were $0.5 \mathrm{~ms}$ and $1.4 \mathrm{~ms}$ in the low fueling rate cases, in contrast to $3.05 \mathrm{~ms}$ and $2.7 \mathrm{~ms}$ in the high fueling rate cases, indicating an earlier development of ignition kernel in the low fueling rate cases, consistent with observations made in previous studies [8]. Table 1 also shows that $t_{2}$ were $3.5 \mathrm{~ms}$ and $3.0 \mathrm{~ms}$ in the low fueling rate cases, in contrast to $5.6 \mathrm{~ms}$ and $5.2 \mathrm{~ms}$ in the high fueling rate cases, indicating an earlier transition to stable flame in the low fueling rate cases. Note that under the definition of $t_{2}$ used in this work, 1) the transition to stable flame occurred before the igniters stopped discharging in the low fueling rate cases and after in the high fueling rate cases, and 2) once the ignition kernel was initiated, the transition time to stable flame (i.e., $t_{2}-t_{1}$, last column in Table 1 ) was not necessarily shorter for the low fueling rate than for the high fueling rate cases.

In summary, this section described the experimental setup to record the development of ignition kernel and transition into stable flame from either different angular orientations and explained the nature of the images captured by this setup. This experimental setup illustrates some of the advantages of FBEs. The FBEs are flexible and of a relatively small footprint, so they circumvent the requirement for direct line-of-sight optical access and greatly facilitate the measurement campaign. Of course, there are also drawbacks of using the FBEs. For example, the resolution of the images is degraded when transmitted through the FBEs, and the FBEs attenuate the signal. Detailed measurements of such degradation and attenuation are provided in [30]. Based on the images provided by the FBEs, the subsequent sections describe the 3D reconstruction of the combustion and the inference of 3D flame properties. 


\section{3D tomography reconstruction and validation}

This section provides a brief summary of the algorithm for the 3D tomography reconstruction; more detailed discussions can be found in [27, 28, 35]. Tomography reconstruction can be summarized into two steps. First, a cubical measurement volume of a length of $64 \mathrm{~mm}$ was defined based on the field of view captured by all 8 FBEs. This volume encompassed the region of interest in the cavity (as illustrated by the 2D projections shown in Figure 2 and Figure 3). In the second step, the measurement volume was discretized into $128 \times 128 \times 128\left(\sim 2 \times 10^{6}\right)$ Cartesian voxels (resulting in a nominal spatial resolution of $0.5 \mathrm{~mm}$ in each direction). The goal of the tomography reconstruction was then to solve for the $3 \mathrm{D}$ distribution of the chemiluminescence emissions within these voxels based on the 8 projection measurements provided by the FBEs. The spatial resolution of the 3D reconstruction depends on both the quantity and quality of the cameras used, as detailed in [26]. The use of more cameras provides projection measurements on more pixels to enable a finer discretization (assuming no physical constraints such as optical access and physical space), and the use of better cameras provides projection measurements with higher signal-to-noise ratio to enable reconstruction with enhanced fidelity. The algorithm used here to solve for the 3D distribution was a modified variation of the Algebraic Reconstruction Technique (ART) [36]. The major modifications included the use of simulated annealing to improve the reconstruction $[37,38]$ and the use of geometrical ray-tracing [27, 39] and Monte Carlo simulation [40, 41], so that the inversion can be performed on projections gathered from an arbitrary experimental setup (i.e., not restricted to the so-called parallel-beam projections). The tomographic algorithm used here has been validated via numerical simulations [27] and controlled experiments in both non-reactive [31] and reactive flows [26, 27].

Figure 4 shows two sample 3D reconstructions of the flame at $t=0.8$ and $10 \mathrm{~ms}$ of low fueling rate case 1 from the reconstruction process outlined above. As shown in Table $1, t_{1}$ and $t_{2}$ for this case were $0.5 \mathrm{~ms}$ and $3.5 \mathrm{~ms}$, respectively. Hence, the results at $t=0.8 \mathrm{~ms}$ (Figure 4a) corresponds to the stage where the ignition kernel was developing and transitioning into a stable flame, and the results at $t=10 \mathrm{~ms}$ (Figure $4 \mathrm{~b}$ ) is the stage where the transition was completed. Figure $4 \mathrm{a}$ and Figure $4 \mathrm{~d}$ show a 3D rendering of the envelope of the flame extracted from the 3D reconstruction. A detailed description of the extraction method will be discussed in the next 
paragraph. In Figure 4a, the two separate sparks and an ignition kernel were observed, while in Figure $4 \mathrm{~d}$ a stable flame occupying almost the entire cavity was observed. To facilitate the interpretation of the 3D reconstructions, Figure $4 \mathrm{~b}, \mathrm{c}$, e, and f show the corresponding projection measurements from the near top view (captured by FBE input 5 at $89^{\circ}$ ) and the side view (captured by FBE input 8 at $0^{\circ}$ ). Comparison between the $3 \mathrm{D}$ reconstructions and the projection measurements illustrates the validity and utility of the 3D measurements. For instance, Figure 4a reproduced the separate sparks and the ignition kernel as seen from the projections. However, each projection only provided partial information: the top view shown in Figure $4 \mathrm{~b}$ captured the two sparks at this particular moment but not the kernel (due to restrictions in optical access), and the side view shown in Figure 4c capture one perspective of the kernel but the sparks were overlapping. For the stable flame, the 3D rendering shown in Figure $4 \mathrm{~d}$ is close to the view from the top, hence the rendering appears similar in both overall shape and in detailed structures compared to the projection measured from the top view (i.e., Figure 4e). The side view shown in Figure $4 \mathrm{f}$ is unable to reveal the $3 \mathrm{D}$ structure of the flame. Besides such visual comparison, the 3D tomographic reconstruction has also been validated via other methods in our past work, for both numerical simulations [27] to controlled flames [42].

The results shown in Figure 5 further illustrate the 3D reconstructions and the extraction of the flame envelope from them. In this work, the flame envelope was determined based on the 3D reconstructions (e.g., those shown in Figure 4a and d) using a thresholding method. The method consisted of two steps. First, the average background level was determined from the raw projection measurements. Second, the background level was applied as the threshold value to the 3D reconstructions to determine the flame envelope. More specifically, the 3D reconstructions were separated into two zones by an iso-surface (i.e., the flame envelope): a flame zone with chemiluminescence signal larger than the threshold value and a non-flame zone otherwise. This method was designed to extract the outer edge of the flame to facilitate comparison with 2D lineof-sight averaged chemiluminescence measurements. Based on the 3D envelope extracted, 2D flame edges can be obtained at an arbitrary location and orientation. Figure 5 shows the 2D flame edges extracted at two difference times $(t=0.6 \mathrm{~ms}$ and $0.8 \mathrm{~ms})$ for low fueling rate case 1 . The 2D flame edges were extracted from the $3 \mathrm{D}$ reconstruction at $Z=10 \mathrm{~mm}$. Figure $5 \mathrm{a}$ shows that the $2 \mathrm{D}$ edges extracted overlapped with a plot of the $3 \mathrm{D}$ reconstruction at $Z=10 \mathrm{~mm}$. The purpose of the comparison shown in Figure 5a was to show how well the edge extraction 
algorithm can extract the outer edge of the flame from the 3D reconstruction. The comparison in Figure 5a shows that the edges extracted from the thresholding method faithfully tracked the outer envelope of the flame as mentioned above. Figure $5 \mathrm{~b}$ shows the extracted $2 \mathrm{D}$ edges and overlapped with projection measurements obtained from FBE 8 . FBE 8 was placed at a view angle of $0^{\circ}$, and therefore its projection measurements were along the same orientation as the $2 \mathrm{D}$ edges extracted here (i.e., along the $Z$ axis). The purpose of the comparison shown in Figure $5 \mathrm{~b}$ was to show the difference between the 3D reconstructions and the line-of-sight-averaged chemiluminescence measurements. It can be seen that the 2D flame edges encompass only some part of the projection measurements, because the 3D nature of the flame and the line-of-sight nature of the projection measurements.

The above thresholding method was also applied to identify the signal emitted by the sparks so that the sparks can be separated from the flame as shown in Figure 6. Figure 6a shows that the envelopes of both sparks (extracted from the 3D reconstruction using the above thresholding method), overlapped with a plot of the $3 \mathrm{D}$ reconstruction at $Y=5 \mathrm{~mm}$, again illustrating the fidelity of the method in extracting an outer envelope for the sparks. Figure $6 \mathrm{~b}$ shows the envelopes overlapped with projection measurements from the same orientation. The ability to identify the sparks as illustrated here allows us to analyze the flames only without the interference of the sparks.

\section{3D properties of ignition kernel}

3D properties of the ignition kernel, including volume, surface area, shape, and velocity, can provide a wealth of information to elucidate the ignition process in the supersonic combustor. For instance, 3D volume and surface area can reveal the development and growth of the ignition kernel, and the 3D shape and velocity can reveal how the ignition kernel is sheared by and interacts with the flow. Therefore, this section describes the extraction of such 3D properties based on the 3D reconstructions discussed in Section 3. By performing tomographic reconstruction on the measured projections frame by frame, 3D volume, surface area, sphericity, and three-component velocity of the flame kernels were extracted to illustrate the spatiotemporal dynamics of the supersonic combustor. 
Figure 7 shows the volume of the flame calculated from the 3D reconstructions for the low fueling rate case 1 (panel a) and high fueling rate case 1 (panel b), respectively. Results obtained on the other two cases (i.e., low fueling rate case 2 and high fueling rate case 2) were similar and led to the same observations and conclusions discussed in the rest of this paper. Therefore, only results obtained in low fueling rate and high fueling rate case 1 are shown here. The contributions from the sparks were removed using the methods discussed. Both the low fueling rate and high fueling rate results show two distinct stages: 1) the ignition stage during which the volume of the flame grew rapidly, and 2) the stable combustion stage during which the volume remained relatively constant. To quantify the transition time (i.e., $t_{2}$ introduced in Section 2) from the ignition stage to the stable combustion stage, we defined $t_{2}$ as the time that the flame volume reached the average of the stable stage. For instance, for the results shown in Figure 7 a (low fueling rate case 1 ), the average volume (during $t=5 \mathrm{~ms}$ to $10 \mathrm{~ms}$ ) was calculated first as shown, and the time when the flame volume first reached this value was then defined as $t_{2}$ (which was determined to be $3.5 \mathrm{~ms}$ in this case). For the results shown in Figure $7 \mathrm{~b}$ for the first high fueling rate case, $t_{2}$ was determined to be $5.6 \mathrm{~ms}$ in this case. The transition times for other cases were summarized in Table 1. These results show that the ignition kernel initiated earlier and transitioned into a stable flame earlier under low fueling rate conditions than under high fueling rate conditions, which is in agreement with past observations [8].

Figure 8 examines the ignition processes more closely before the kernel transitioned to stable flames. The variation of the spark volume is also plotted. Note that the volume of the spark emissions is expressed on a linear scale because it varied little, while that of the ignition kernel is on a logarithm scale because it varied across two orders of magnitude. As can be seen from Figure 8, the volumes of the sparks varied in a narrow range of 20 to $30 \mathrm{~mm}^{3}$. The volume formed by the emissions of spark 2 was consistently larger than that of spark 1 . The spark plugs were identical, and the spark-plug gaps were identical between the center high voltage electrode and the surrounding ground. However, the spark may be elongated beyond that distance because of the flow, resulting in difference discharges as seen here. The sudden drop of the sparks' volumes (near $t=3.8 \mathrm{~ms}$ for low fueling rate case 1 shown in Figure 8a, and near $t=3.75 \mathrm{~ms}$ for high fueling rate case 1 shown in Figure 8b) indicates the time the sparks stopped discharging. As discussed earlier in Section 2 and shown here, we were able to reliably determine the ignition kernel only in some cases. Major factors in the reliable identification of the ignition kernel 
include the size and signal (both must be sufficiently large), so that it can be captured by the camera and separated from the spark emission unambiguously. For the two cases shown here, the kernel can be reliably determined at $t_{1}=0.5 \mathrm{~ms}$ for the low fueling rate case, as shown in Figure $8 \mathrm{a}$, and $t_{1}=3.05 \mathrm{~ms}$ for the high fueling rate case, as shown in Figure 8b, where the kernel's size was on the order of tens of $\mathrm{mm}^{3}$. More sensitive measurements (e.g., using intensified cameras and/or imaging in another spectral range) are needed to resolve the development of the ignition kernel at an earlier stage. Note the FBEs can be used with intensifiers too, and was tested in the supersonic combustor. However, there was only one high-speed intensifier available to us at the time these experiments were conducted. Also note that the exclusion of the spark emission from the flame emission was important when the sparks were first observed, as the volume of the spark emission was on the same order of magnitude as the ignition kernel. As the ignition kernel grew, such exclusion became less important.

Figure 9 shows the surface area of the spark emissions and the ignition kernel for the low fueling rate case 1 (panel a) and high fueling rate case 1 (panel b). Similar observations and insights about the ignition processes can be obtained from these results as those from the volume results. With 3D volume and surface area determined, a shape factor can be calculated to quantify the irregularity of the flame. This work used the sphericity as defined below to quantify the irregularity of a 3D object:

$$
\text { Sphericity }=\frac{\pi^{\frac{1}{3}}(6 V)^{\frac{2}{3}}}{A}
$$

where $V$ is the volume of the object and $A$ its surface area. This definition essentially quantifies the irregularity of a $3 \mathrm{D}$ object relative to a sphere. Based on this definition, a sphere has a sphericity of 1 , and all non-spherical objects have a value less than 1 . The smaller the sphericity, the "more irregular" the object is compared to a sphere. For example, a hemisphere has a sphericity of 0.84 , and an infinitely long and slender cylinder has a sphericity approaching 0 . Figure 10 shows the sphericity of the ignition kernel and the sparks for the low fueling rate and high fueling rate case, based on the volume and surface area results shown in Figure 8 and Figure 9. The shape of the ignition kernel was affected strongly by its interaction with the flow, e.g., due to stretching in the shear layer. The results in Figure 10 clearly show dynamics due to the 
complicated interactions between the flame and the flow and that the shape of the ignition kernel was highly irregular. Figure 10 shows that the sphericity of the ignition kernel varied between 0.7 and 0.3 for both the low fueling rate and high fueling rate cases (e.g., at the four data points highlighted with triangles and squares). In contrast, the sphericity of the sparks remained more stable within a range of 0.8 to 1 , indicating a more stable and regular shape due to several possible reasons. The plasmas formed by the sparks were further away from the shear layer than the ignition kernel, and therefore were subject to weaker interactions with flow. Also, the emissions from the plasmas were sufficiently strong to saturate a portion of the projection measurements, causing ambiguity in their reconstructions. These observations were confirmed by the $3 \mathrm{D}$ reconstructions shown in Figure $4 \mathrm{a}$ and $\mathrm{b}$.

To further illustrate the interactions between the flame and flow, the 3D and 3-component (3D3C) velocities of the ignition kernel were estimated based on the 3D measurements. Here, the 3D3C velocity of the ignition kernel was estimated based on the movement of the centroid of the kernel between 2 consecutive frames of 3D measurements (i.e., during a time interval of $50 \mu$ s). More specifically, based on the 3D reconstructions obtained above, the 3D coordinates of the centroid of the ignition kernel were determined frame by frame. The centroid was calculated (or defined) as the weighted averaging of the coordinates of all voxels in the flame zone, and the weighting factor was the chemiluminescence intensity of each voxel. With the $3 \mathrm{D}$ coordinates of the centroid determined, the movement of the centroid between two consecutive frames in all three spatial directions was calculated. Lastly, the 3D3C velocity vectors of the ignition kernel were obtained by dividing such movement by the frame-to-frame interval (i.e., $50 \mu \mathrm{s}$ ).

The 3D3C velocity vector obtained from the above procedure are shown in Figure 11, Figure 12, and Figure 13 for a low and a high fueling rate case during the ignition stage. Figure 11 shows the 3D3C velocity vectors, Figure 12 shows the magnitude of the velocity vector, and Figure 13 shows the $2 \mathrm{D}$ projections of the $3 \mathrm{D} 3 \mathrm{C}$ velocity in the top and side views to facilitate the interpretation of the results and the comparison versus PIV measurements. The overall pattern of the flow field revealed by the velocity vectors shown in Figure 11 and Figure 12 and magnitude of the velocity vectors shown in Figure 13 are in general agreement with previous PIV measurements [8]. However, a quantitative comparison between the results reported here and the PIV is difficult, primarily due to i) the mismatch of the dimensionality between PIV and 
the $3 \mathrm{D}$ results and ii) approximations invoked in the estimation of the $3 \mathrm{D} 3 \mathrm{C}$ velocity in this work. As mentioned above, the velocity was calculated by the movement of the centroid of the kernel. As a result, the uncertainty in this calculation depended on the size of the kernel, and the uncertainty increased as the kernel grew in size. To help illustrate the uncertainty caused by the size of the kernel, a critical kernel size and its corresponding time was identified in our calculation. Before such critical time, the kernel's size was smaller than the critical value so that there was no spatial overlap of the kernel between two consecutive measurements. As a result, the uncertainty in the calculation of velocity was relatively small before the kernel's size reached this critical time. After such critical time, the kernel's size had grown sufficiently so that the kernel measured between frames overlapped spatially, resulting in an increased uncertainty in the calculation of velocity based on the centroid location. The data points corresponding to such critical time and size were highlighted by a circle in Figure 11, Figure 12, and Figure 13. For instance, for the results shown in Figure 11a, the critical time was determined to be $t=1.5 \mathrm{~ms}$.

From the results shown in Figure 11 and Figure 13, it can be seen that the ignition kernel was first initiated near one of the sparks, and then the kernel moved in a range of $\sim 20 \mathrm{~mm}$ in the $X$ direction, $\sim 10 \mathrm{~mm}$ in the $Y$ direction, and $\sim 30 \mathrm{~mm}$ in the $Z$ direction before it transitioned to a stable flame. Also note that the ignition kernel initiated at a location further away from the sparks under the high fueling rate condition than the low fueling rate condition. Figure 12 shows the magnitude of the velocity vectors. The velocity in either of low fueling rate and high fueling rate cases generally fluctuated in the range from 20 to $100 \mathrm{~m} / \mathrm{s}$, due to the effect of the main flow in the cavity. To better illustrate the movement of the ignition kernel within the cavity, Figure 13 shows the $2 \mathrm{D}$ projection of the $3 \mathrm{D} 3 \mathrm{C}$ velocity vectors for a low fueling rate (panels a and $\mathrm{b}$ ) and high fueling rate case (panels $\mathrm{c}$ and d). The results here show the distance the kernel traveled from frame to frame, plotted in scale with the dimension of the cavity. In both cases, the ignition kernel first appeared when flow moved upstream following the average direction of velocity within the cavity's recirculation bubble, and it required a certain period of time for the chemical reactions to initiate and accumulate enough electronically excited radicals to emit a detectable chemiluminescence signal. After moving farther until about $4 \mathrm{~mm}$ from the front step of the cavity, the ignition kernel then turned back and moved downstream along with the flow in the shear layer. A stable combustion region then developed and anchored near the ramp and fuel injectors. 
Lastly, the 3D3C velocity calculated from the 3D measurements also help to interpret the flame-flow interactions. As mentioned earlier in the discussion of the sphericity in Figure 10, the shape of the kernel reflected the interaction and distortion of the flame with the flow. As a result, the sphericity varied dynamically and in a large range between 0.3 and 0.7 under both the low fueling rate and high fueling rate conditions, as illustrated by the four data points highlighted in the triangles (when the sphericity was $\sim 0.7$ ) and squares (when the sphericity was $\sim 0.3$ ) in Figure 10. These four data points were also highlighted in Figure 11 and Figure 13. As can be seen from the velocity results, especially the side view of velocity shown in Figure 13b and Figure 13d, the ignition kernel moved through a recirculation zone during the time marked by the triangles and the time marked by the squares. Due to the intense interactions between the kernel and flow in the recirculating zone, the shape of the kernel varied dramatically, as reflected in the large change of the sphericity reported in Figure 10.

\section{Summary and Conclusions}

The ignition process in the cavity flameholder of a Mach-2 crossflow was investigated using 3D measurements at $20 \mathrm{kHz}$, with a focus on the detailed spatiotemporal dynamics of the initiation of the ignition kernel and its transition into a stable flame. The measurements were performed based on tomographic chemiluminescence implemented with fiber-based endoscopes (FBEs). Projection measurements of chemiluminescence images from the cavity were simultaneously captured from 8 orientations using 2 customized FBE bundles at $20 \mathrm{kHz}$. The measured projections were then fed into a tomographic algorithm as inputs to obtain 3D reconstructions. Based on the 3D reconstructions, other flame and flow properties were extracted to study the ignition processes, including 3D volume, surface area, sphericity, and 3D3C velocity of the ignition kernel. The 3D volume and surface area measurements clearly illustrated the two stages in the supersonic combustion process: an ignition stage during which the ignition kernel grew rapidly, and a stable combustion stage during in which volume and surface area varied little. The 3D volume and surface area measurements also enabled the determination of a transition time to quantify these two stages. Based on the 3D volume and surface area, the sphericity of the kernel was also determined to quantify its irregular shape. When combined with the 3D3C velocity, the sphericity illustrates the flame-flow interactions of the cavity flameholder. The results obtained in this work demonstrate the utility of the 3D diagnostics to resolve the detailed 
spatiotemporal dynamics of high-speed propulsion devices and also of FBEs to facilitate the implementation of such diagnostics in practical systems. Our ongoing work includes the extension of the 3D tomographic measurements to other signal generation processes (such as volumetric laser induced fluorescence) and a combination of the tomographic technique with other diagnostics (such as simultaneous tomographic chemiluminescence and PLIF).

\section{Acknowledgement}

This work is supported by the U.S. Air Force Office of Scientific Research (AFOSR) with Dr. Chiping $\mathrm{Li}$ as the technical monitor. Development of the imaging processing and analysis algorithms used here was supported by an NSF award (Award CBET 1156564). Lin Ma is also grateful for a 2014 U.S. Air Force Summer Faculty Fellowship. 


\section{References}

1. C. C. Rasmussen; S. K. Dhanuka; J. F. Driscoll, Proceedings of the Combustion Institute 31 (2007) 2505-2512 10.1016/j.proci.2006.08.007.

2. O. Timothy; D. C. Campbell; T. Chung-Jen; H. KuangYu, in: 52nd Aerospace Sciences Meeting, American Institute of Aeronautics and Astronautics: 2014.

3. Y. Vigor; L. Jian; C. Jeong Yeol; L. Kuo-Cheng, in: 48th AIAA Aerospace Sciences Meeting Including the New Horizons Forum and Aerospace Exposition, American Institute of Aeronautics and Astronautics: 2010.

4. Y. Vigor; L. Jian; C. Jeong Yeol; L. Kuo-Cheng, in: 48th AIAA Aerospace Sciences Meeting Including the New Horizons Forum and Aerospace Exposition, American Institute of Aeronautics and Astronautics: 2010.

5. Y. Moule; V. Sabelnikov; A. Mura, Combustion and Flame 161 (10) (2014) 2647-2668 10.1016/j.combustflame.2014.04.011.

6. W. R; K. R; M. J, in: 28th Joint Propulsion Conference and Exhibit, American Institute of Aeronautics and Astronautics: 1992.

7. M. B. Sun; C. Gong; S. P. Zhang; J. H. Liang; W. D. Liu; Z. G. Wang, Experimental Thermal and Fluid Science 43 (0) (2012) 90-96 http://dx.doi.org/10.1016/j.expthermflusci.2012.03.028.

8. S. G. Tuttle; C. D. Carter; K.-Y. Hsu, Journal of Propulsion and Power 30 (3) (2014) 576-591 10.2514/1.B34974.

9. T. M. Ombrello; C. D. Carter; C.-J. Tam; K.-Y. Hsu, Proceedings of the Combustion Institute (35) (2015) http://dx.doi.org/10.1016/j.proci.2014.07.068.

10. R. M. Spearrin; C. S. Goldenstein; I. A. Schultz; J. B. Jeffries; R. K. Hanson, Applied Physics BLasers and Optics 117 (2) (2014) 689-698 10.1007/s00340-014-5884-0.

11. O. B. Sean; S. Ingo; N. Andrew; B. Russell; M. Neil; H. Frank, in: AIAA/CIRA 13th International Space Planes and Hypersonics Systems and Technologies Conference, American Institute of Aeronautics and Astronautics: 2005.

12. M. Ryan; M. Gruber; C. Carter; T. Mathur, Proceedings of the Combustion Institute 32 (2) (2009) 2429-2436 http://dx.doi.org/10.1016/j.proci.2008.06.209.

13. E. Lazar; G. Elliott; N. Glumac, Aiaa Journal 46 (12) (2008) 2987-2997 10.2514/1.32835.

14. C. Carter; S. Hammack; T. Lee, Appl. Phys. B 116 (3) (2014) 515-519 10.1007/s00340-0145899-6.

15. F. Fuest; M. J. Papageorge; W. R. Lempert; J. A. Sutton, Optics Letters 37 (15) (2012) 3231 3233

16. S. Roy; J. D. Miller; M. N. Slipchenko; P. S. Hsu; J. G. Mance; T. R. Meyer; J. R. Gord, Optics Letters 39 (22) (2014) 6462-6465 10.1364/ol.39.006462.

17. I. A. Schultz; C. S. Goldenstein; J. B. Jeffries; R. K. Hanson; R. D. Rockwell; C. P. Goyne, Journal of Propulsion and Power 30 (6) (2014) 1551-1558 10.2514/1.b35219.

18. L. Ma; X. Li; S. T. Sanders; A. W. Caswell; S. Roy; D. H. Plemmons; J. R. Gord, Optics Express 21 (1) (2013) 1152-1162 10.1364/OE.21.001152.

19. V. A. Miller; V. A. Troutman; R. K. Hanson, Measurement Science \& Technology 25 (7) (2014) 10.1088/0957-0233/25/7/075403.

20. K. Y. Cho; A. Satija; T. L. Pourpoint; S. F. Son; R. P. Lucht, Applied Optics 53 (3) (2014) 316326 10.1364/ao.53.000316.

21. Q. Gao; C. Ortiz-Duenas; E. K. Longmire, Experiments in Fluids 54 (12) (2013) 10.1007/s00348-013-1625-0.

22. I. Boxx; C. D. Carter; W. Meier, Experiments in Fluids 55 (8) (2014) 10.1007/s00348-014-1771$\mathrm{Z}$.

23. B. Coriton; A. M. Steinberg; J. H. Frank, Experiments in Fluids 55 (6) (2014) 10.1007/s00348014-1743-3. 
24. M. R. Gruber; A. S. Nejad, Journal of Propulsion and Power 11 (5) (1995) 1080-1083 10.2514/3.23940.

25. X. Li; L. Ma, Optics Express 22 (4) (2014) 4768-4778 10.1364/OE.22.004768.

26. X. Li; L. Ma, Combustion and Flame 162 (3) (2015) 642-

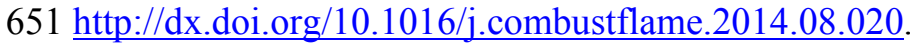

27. W. Cai; X. Li; F. Li; L. Ma, Optics Express 21 (6) (2013) 7050-7064 10.1364/OE.21.007050.

28. W. Cai; X. Li; L. Ma, Appl. Opt. 52 (33) (2013) 8106-8116 10.1364/AO.52.008106.

29. M. Kang; Y. Wu; L. Ma, Combustion and Flame (0)) http://dx.doi.org/10.1016/j.combustflame.2014.06.002.

30. M. Kang; Q. Lei; L. Ma, Appl. Opt. 53 (26) (2014) 5961-5968 10.1364/AO.53.005961.

31. Q. Lei; Y. Wu; H. Xiao; L. Ma, Appl. Opt. 53 (28) (2014) 6389-6398 10.1364/AO.53.006389.

32. M. R. Gruber; J. M. Donbar; C. D. Carter; K. Y. Hsu, Journal of Propulsion and Power 20 (5) (2004) 769-778 10.2514/1.5360.

33. W. K. Min; L. Xuesong; M. Lin, in: 52nd Aerospace Sciences Meeting, American Institute of Aeronautics and Astronautics: 2014.

34. M. Kang; X. Li; L. Ma, Proceedings of the Combustion Institute 35 (3) (2015) 3821-3828

35. J. Floyd; P. Geipel; A. M. Kempf, Combustion and Flame 158 (2) (2011) 376$391 \mathrm{http}: / / \mathrm{dx}$. doi.org/10.1016/j.combustflame.2010.09.006.

36. R. Gordon; R. Bender; G. T. Herman, Journal of Theoretical Biology 29 (3) (1970) 471$481 \mathrm{http} / /$ dx.doi.org/10.1016/0022-5193(70)90109-8.

37. W. Cai; L. Ma, Computer Physics Communications 181 (1) (2010) 11$16 \mathrm{http} / / / \mathrm{dx}$. doi.org/10.1016/j.cpc.2009.08.001.

38. L. Ma; L. Kranendonk; W. Cai; Y. Zhao; J. Baba, Journal of Aerosol Science 40 (7) (2009) 588596 http://dx.doi.org/10.1016/j.jaerosci.2009.02.004.

39. W. Cai; L. Ma, Chinese Optics Letters 10 (1) (2012) 012901

40. Y. Zhao; X. Li; L. Ma, Computer Physics Communications 183 (8) (2012) 15881595 http://dx.doi.org/10.1016/j.cpc.2012.02.027.

41. X. Li; L. Ma, Journal of Quantitative Spectroscopy and Radiative Transfer 114 (0) (2013) 157166 http://dx.doi.org/10.1016/j.jqsrt.2012.08.027.

42. W. Xu; A. J. Wickersham; Y. Wu; F. He; L. Ma, Appl. Opt. 54 (9) (2015) 2174-2182 10.1364/AO.54.002174. 


\section{Table}

\begin{tabular}{lccccc}
\hline & $\mathrm{t}_{0}(\mathrm{~ms})$ & $\mathrm{t}_{1}(\mathrm{~ms})$ & $\mathrm{t}_{2}(\mathrm{~ms})$ & $\mathrm{t}_{3}(\mathrm{~ms})$ & $\mathrm{t}_{2}-\mathrm{t}_{1}(\mathrm{~ms})$ \\
\hline Low fueling rate case 1 & 0.00 & 0.50 & 3.50 & 3.80 & 2.75 \\
Low fueling rate case 2 & 0.00 & 1.40 & 3.00 & 4.10 & 1.60 \\
High fueling rate case 1 & 0.00 & 3.05 & 5.60 & 3.75 & 2.45 \\
High fueling rate case 2 & 0.00 & 2.70 & 5.20 & 3.80 & 2.50 \\
\hline
\end{tabular}

Table 1 . Summary of characteristic times, $t_{0}$ to $t_{3}$, for the cases analyzed in this work. 


\section{Figures}

(a)

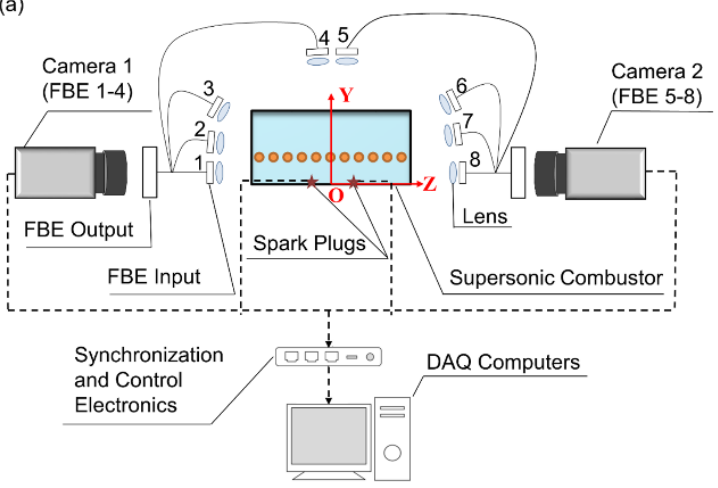

(b)

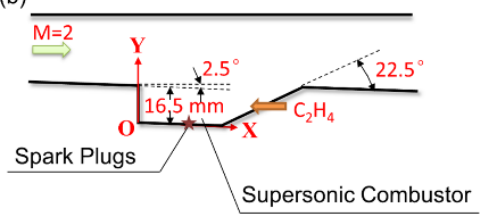

(c)

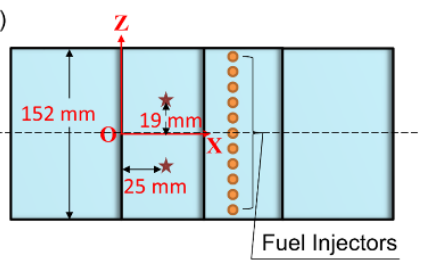

Figure 1. Experimental setup. Panel (a): the overall experimental setup and end view of the wind tunnel (the flow issues into the page). Panel (b): the wind tunnel from the side view. Panel (c): the wind tunnel from the top view.
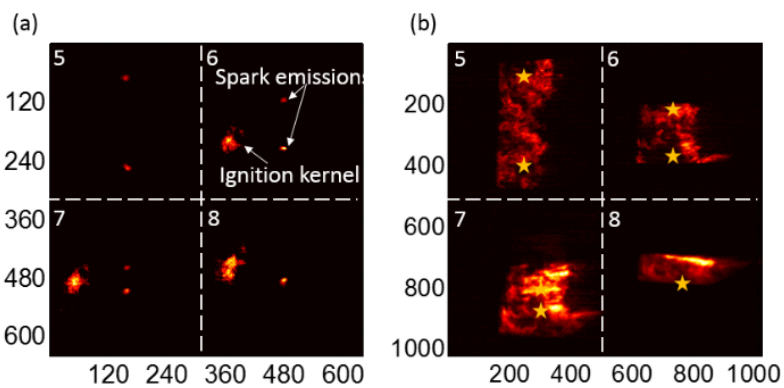

Figure 2. Two frames of sample projections recorded by camera 2 in low fueling rate case (case 1): (a) frame 16 corresponding $t=0.8 \mathrm{~ms}$; (b) frame 80 corresponding $t=10 \mathrm{~ms}$. 


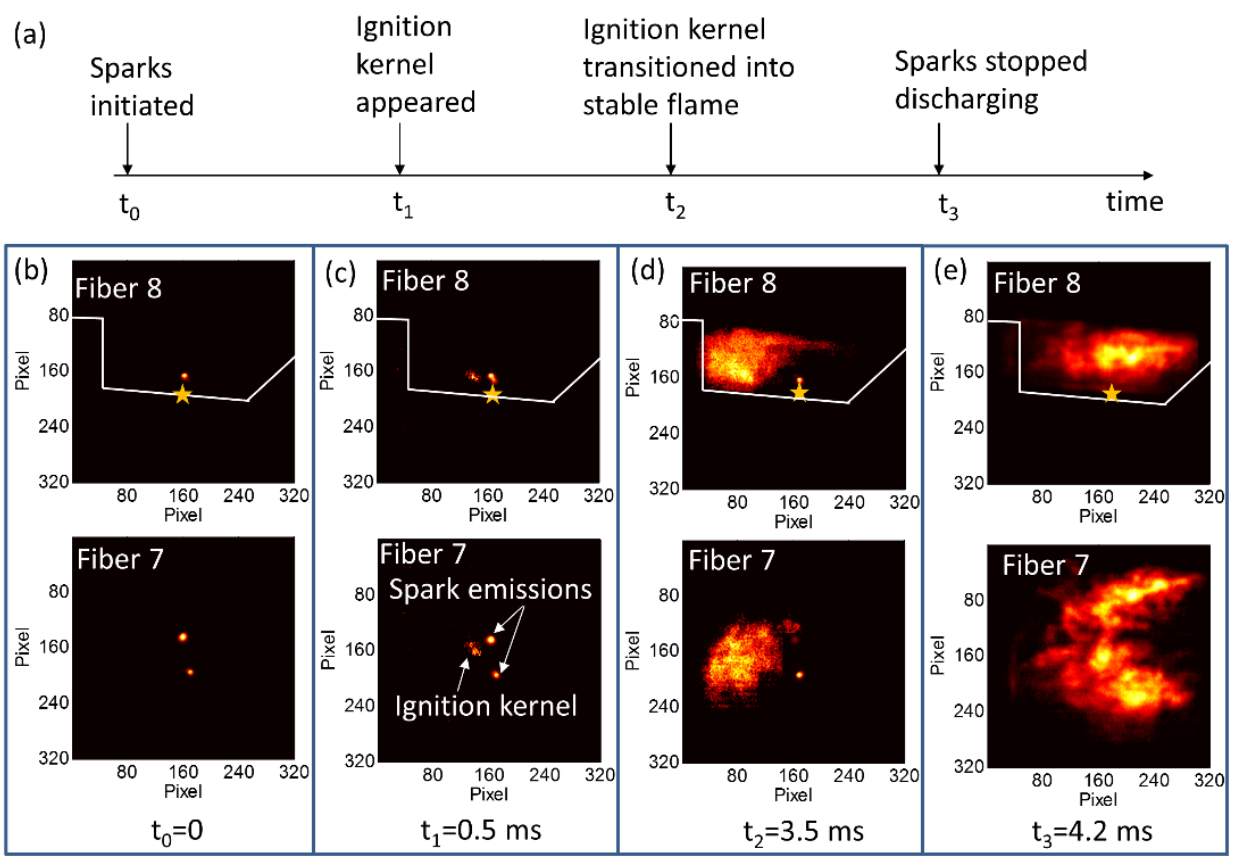

Figure 3. Frames at several characteristic times showing spark and ignition kernels in low fueling rate case 1. Panel (a): definition of the characteristic times; panel (b) frames at $t_{0}$ showing the beginning of spark discharge; panel (c): frames at $t_{1}$ showing the first occurrence of ignition kernel; panel (d): frames at $t_{2}$ showing the transition from ignition kernel into stable flame; panel (e): frames at $t_{3}$ showing the termination of sparks discharge.

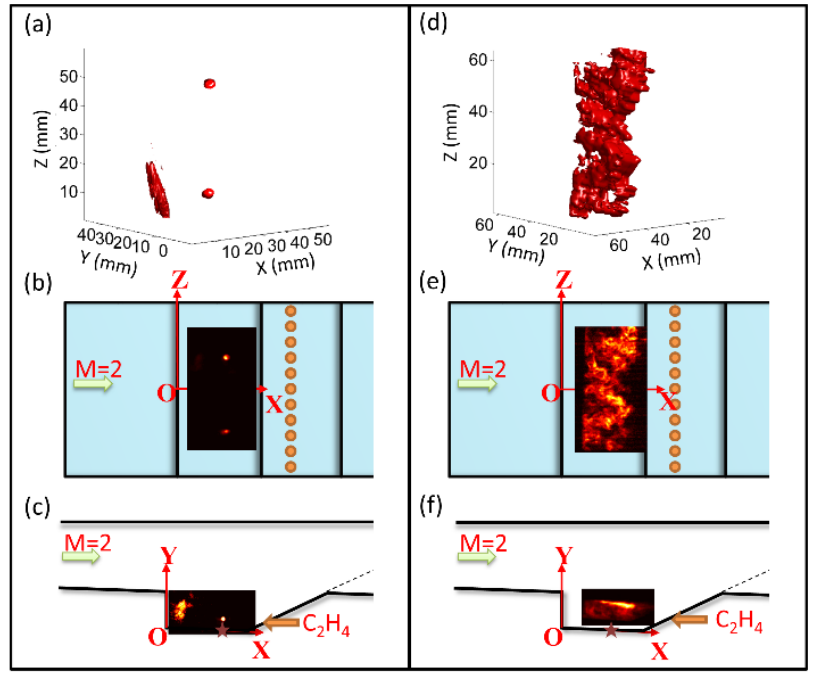

Figure 4. Comparison of 3D reconstruction with projections measured from the top and side for both the ignition phase (panels a, b, and c) and the stable combustion phase (panels d, e, and f). 


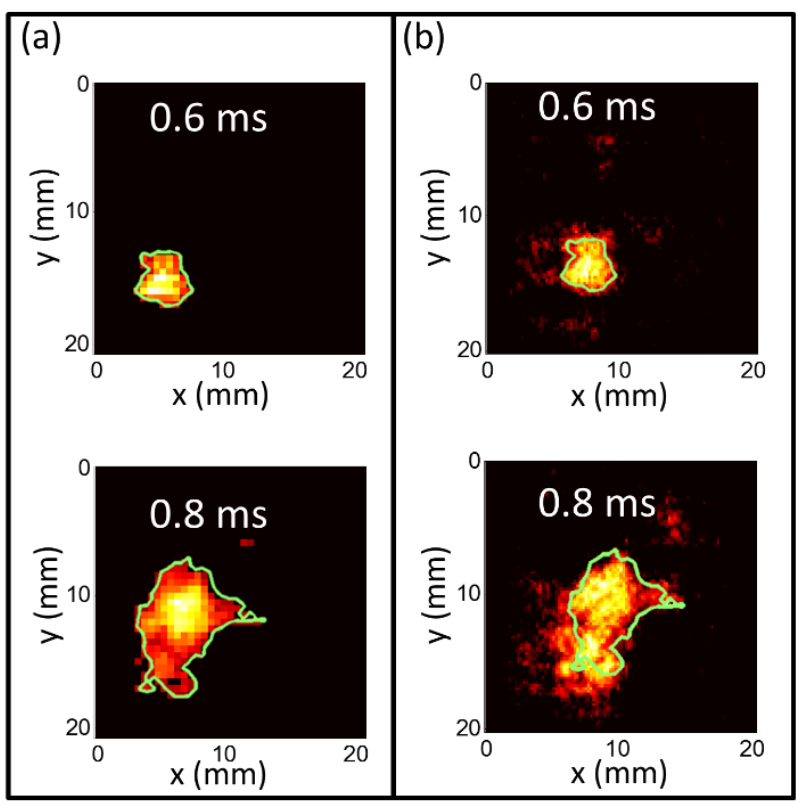

Figure 5. Illustration of the 3D reconstructions of the ignition kernels and the edge extraction method applied on the kernels. Panel a: comparison of a 2D cross-section of the 3D reconstruction with the edges extracted. Panel b: comparison of measured projection with the edges extracted.

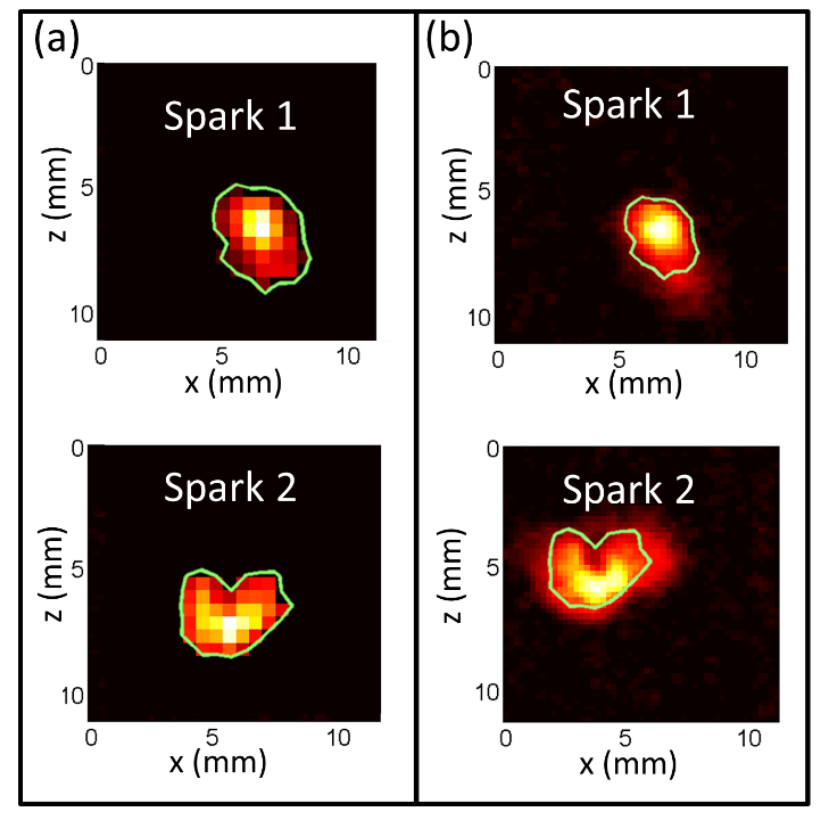

Figure 6. Illustration of the 3D reconstructions of the spark emissions and the application of the edge extraction method on the spark emissions. Panel a: comparison of a 2D cross-section of the 3D reconstruction with edges extracted. Panel $b$ : comparison of a measured projection with the edges extracted. 


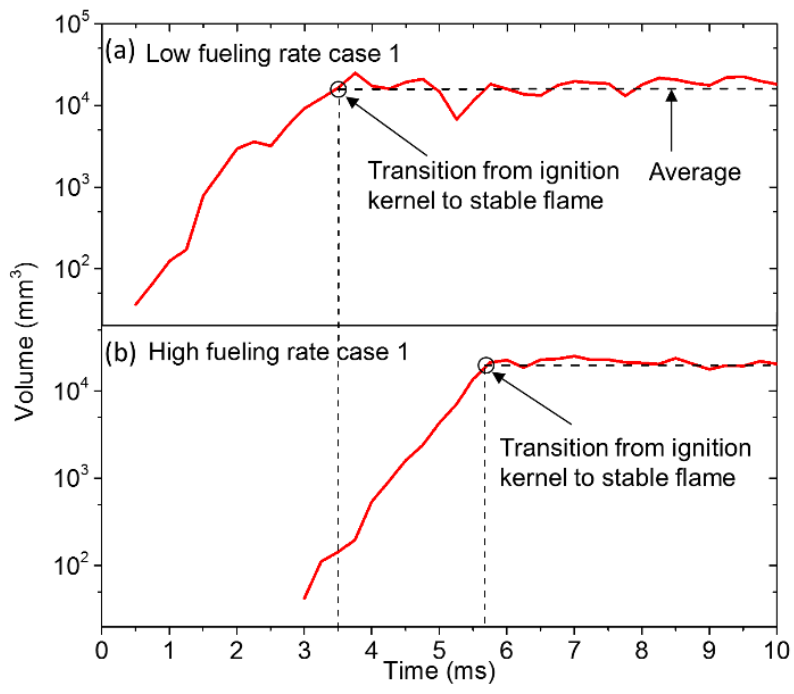

Figure 7. Measured 3D volume for low fueling rate case 1 (panel a) and high fueling rate case 1 (panel b).

(a) Low fueling rate case 1

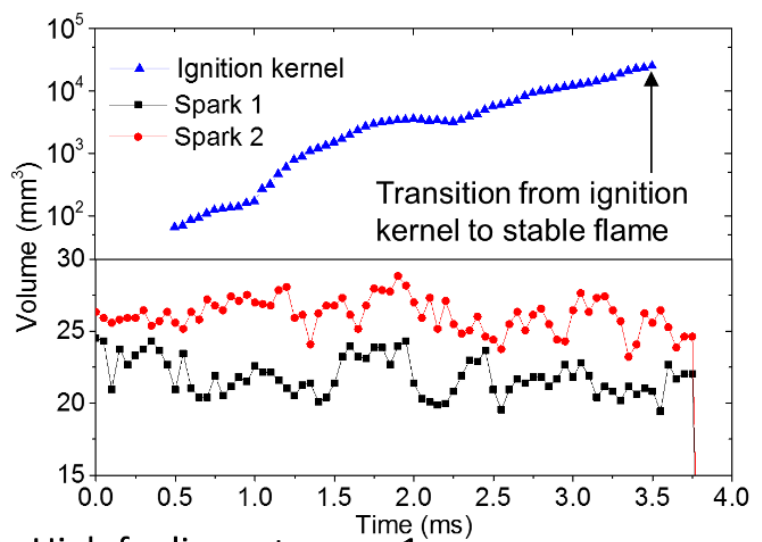

(b) High fueling rate case 1

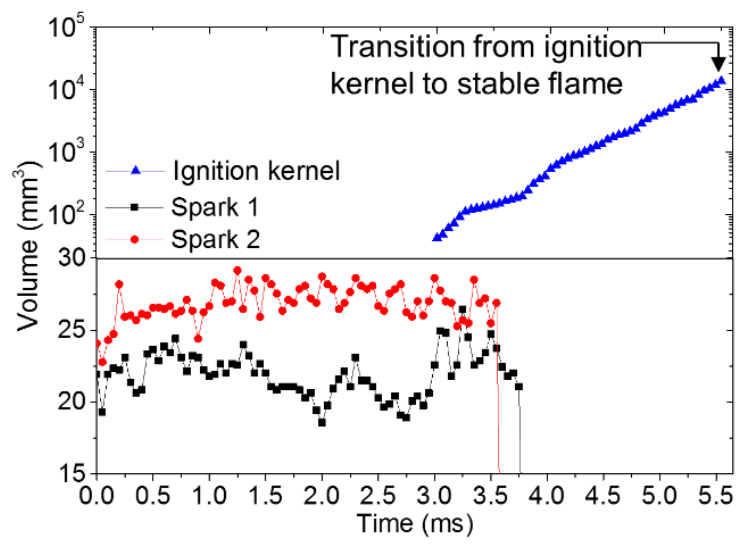

Figure 8. Measured 3D volume of the kernel and spark emissions during the ignition stage. 
(a) Low fueling rate case 1

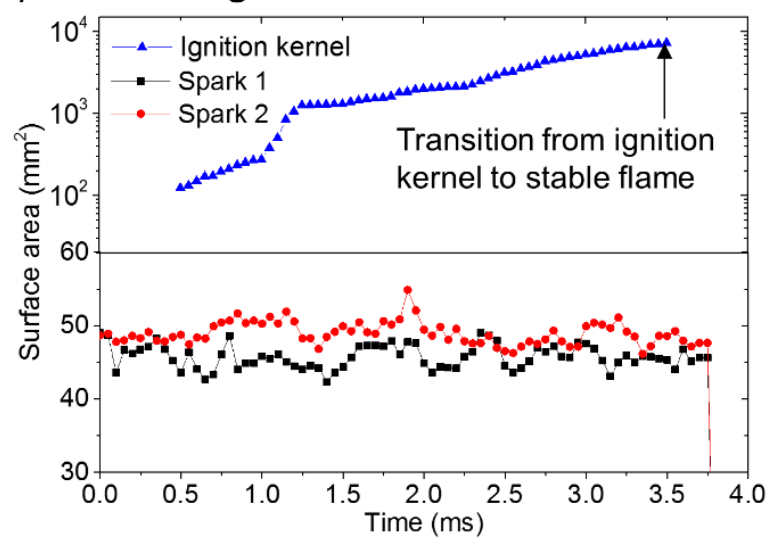

(b) High fueling rate case 1

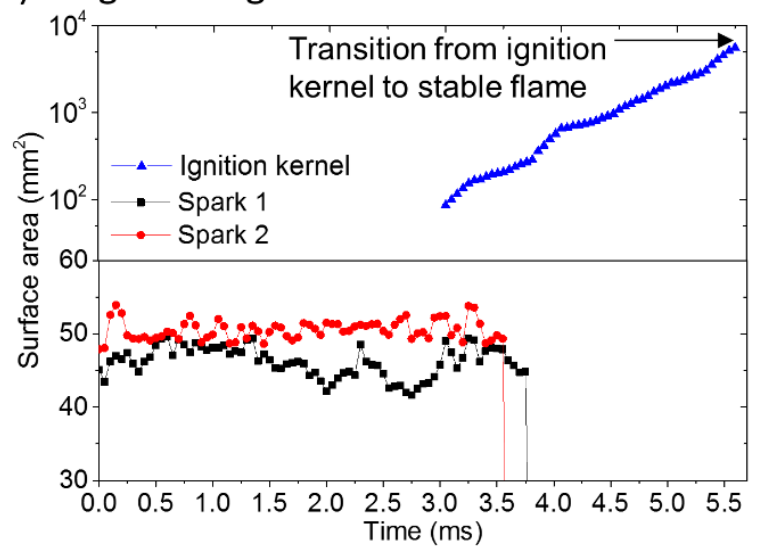

Figure 9. Measured surface area for the kernel and spark emissions during the ignition stage. 
(a) Low fueling rate case 1

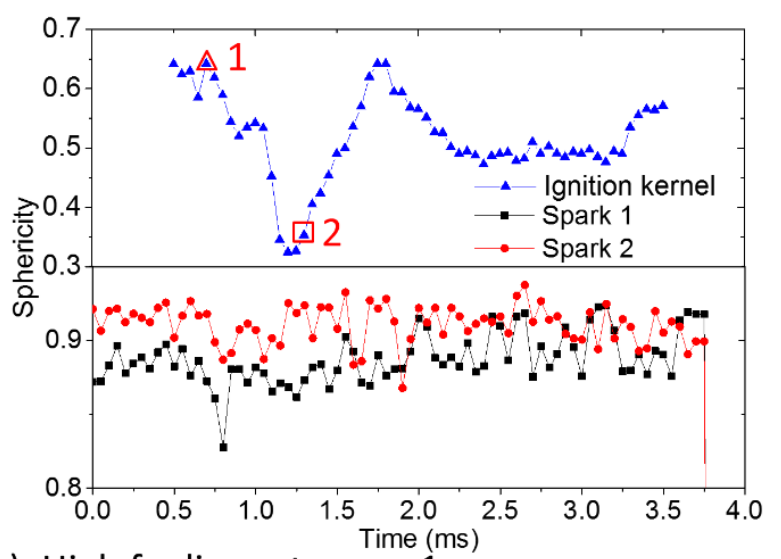

(b) High fueling rate case 1

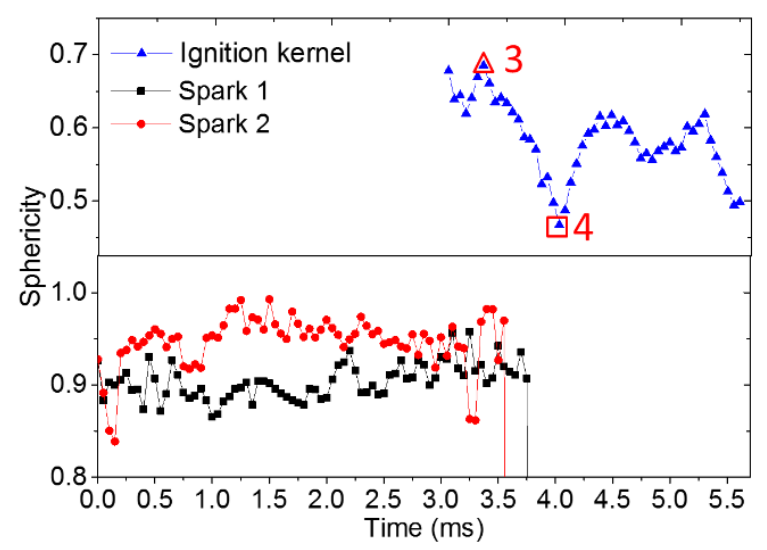

Figure 10. Sphericity of the ignition kernel and spark emissions during the ignition stage. 


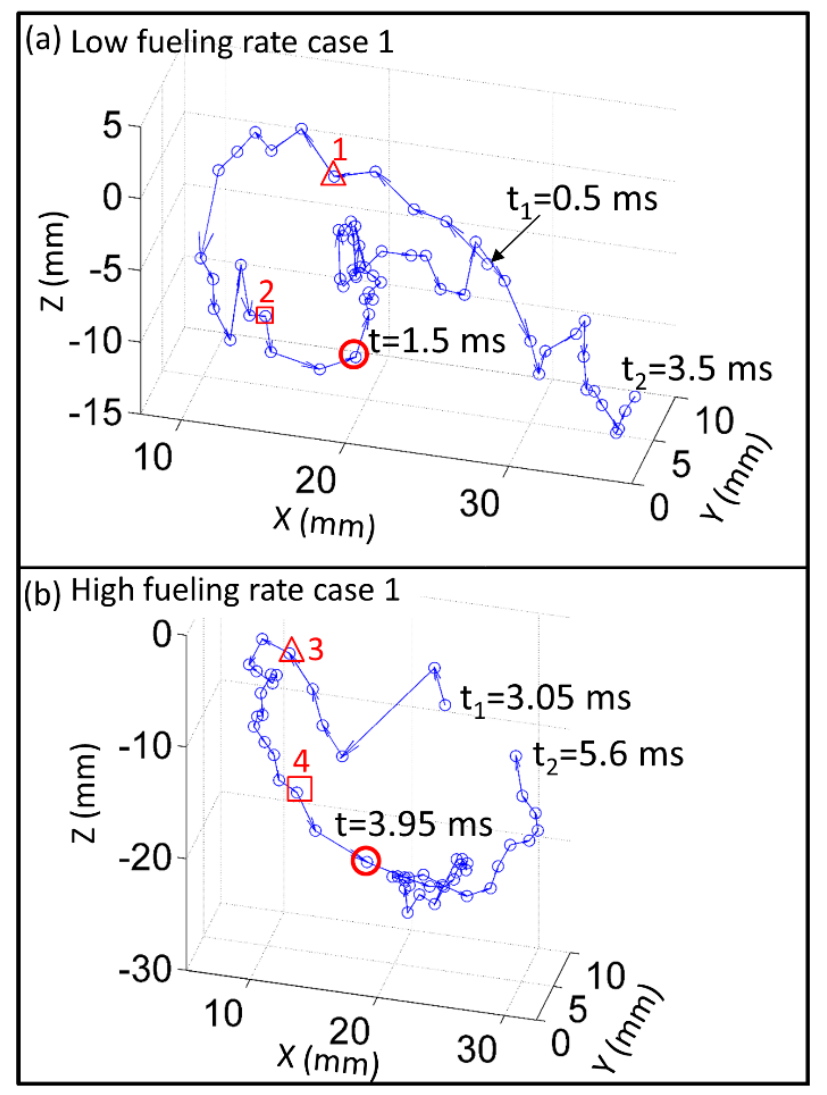

Figure 11. 3D3C velocity vectors extracted from the $3 \mathrm{D}$ reconstructions of the ignition kernel in the low fueling rate case 1 (panel a) and high fueling rate case 1 (panel b). The arrow denotes the magnitude and direction of the velocity vector.

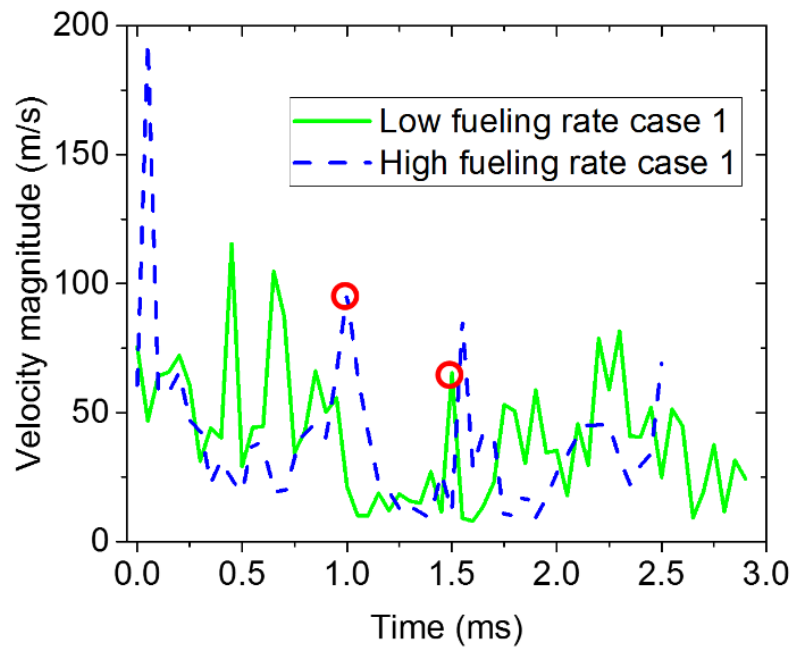

Figure 12. Magnitude of the 3D3C velocity vectors for high fueling rate case 1 and low fueling rate case 2 . 


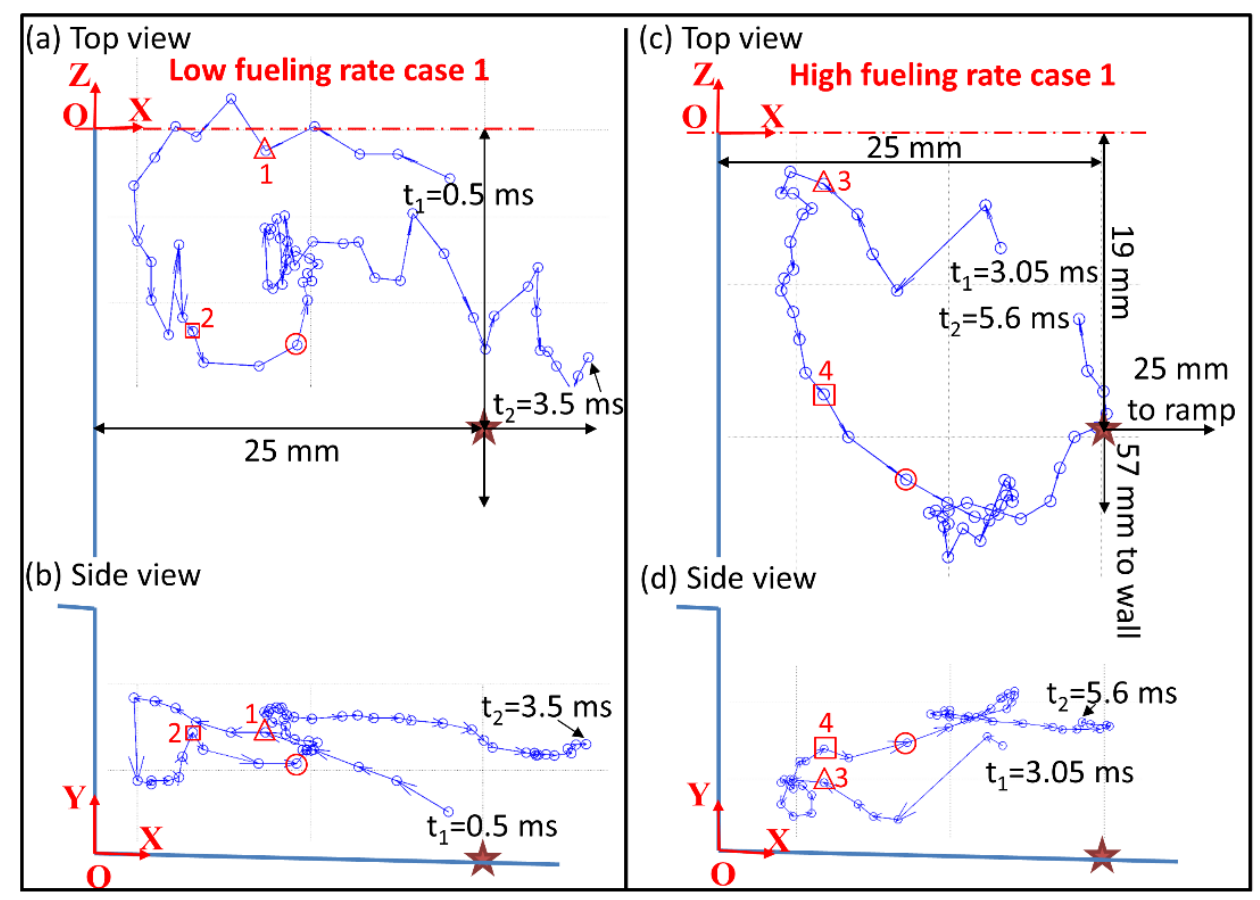

Figure 13. 2D projections of the 3D3C velocity vectors from the top and side views for low fueling rate case 1 (panel a and b) and high fueling rate case 1 (panel c and $d$ ). 